\title{
Fitoacumulacion de arsénico en plantas terrestres del desierto de Chile
}

\author{
Fitoacumulação de arsénico em plantas terrestres do deserto de Chile
}

\author{
Oscar Pablo Díaz \\ Universidad de Concepción - Concepción - Chile \\ Yasna Mariela Tapia \\ Universidad de Chile - Santiago - Chile \\ Rubén Pastene \\ Universidad de Santiago de Chile - Usach - Santiago - Chile
}

\section{Resumen}

El objetivo de este trabajo fue mostrar la capacidad de 4 especies de plantas terrestres: Atriplex atacamensis, Atriplex halimus, Lupinus microcarpus y Tessaria absinthioides para acumular arsénico en suelos agrícolas de la Región de Antofagasta, Chile, para ser utilizadas en fitorremediación y/o revegetación para la recuperación de suelos contaminados, así como también mostrar los efectos del arsénico en las especies $L$. microcarpus, $A$. halimus y $A$. atacamensis a través de bioindicadores de estrés. Los resultados indican que $A$. atacamensis y $A$. halimus resisten la contaminación por arsénico, acumulando el metaloide principalmente en las raíces, pero no en el caso de $L$. microcarpus. Síntomas visuales de toxicidad por arsénico, tales como clorosis y necrosis en las hojas, hojas arrugadas y decrecimiento, fueron observados en la especie $L$. microcarpus.

\section{Resumo}

O objetivo deste trabalho foi mostrar a capacidade de 4 espécies de plantas terrestres: Atriplex atacamensis, Atriplex halimus, Lupinus microcarpus e Tessaria absinthioides em acumular arsênico em solos agrícolas da região de Antofagasta, Chile, para uso na fitorremediação e/ou revegetação para recuperação de solos contaminados, bem como mostrar os efeitos do arsênico sobre as espécies L. microcarpus, $A$. halimus e $A$. atacamensis através de bioindicadores de estresse. Os resultados indicam que $A$. atacamensis e $A$. halimus resistem à contaminação por arsênico, acumulando o metalóide principalmente nas raízes, mas não no caso de L. microcarpus. Sintomas visuais de toxicidade por arsênico, como clorose e necrose de folhas, folhas enrugadas e diminuição de tamanho, foram observados na espécie L. microcarpus.

\section{Palabras clave}

Efectos fitotóxicos. Metal contaminante. Factor de bioconcentración (BCF). Índice de transporte (TI). Región de Antofagasta, Chile.

\section{Palavras-chave}

Efeitos fitotóxicos. Metal contaminante. Fator de bioconcentração (BCF). Índice de transporte (TI). Região de Antofagasta, Chile. 


\section{Introducción}

Las plantas han desarrollado mecanismos altamente eficientes para absorber, translocar y acumular nutrientes (LASAT, 2000), sin embargo, algunos metales y metaloides no esenciales para los vegetales son absorbidos, translocados y acumulados en las plantas debido a que presentan un comportamiento electroquímico similar a los elementos nutritivos esenciales.

Los variados elementos no esenciales que se encuentran en el suelo, tales como el arsénico $(\mathrm{As})$, cadmio $(\mathrm{Cd})$, plomo $(\mathrm{Pb})$, entre otros, pueden someter al vegetal a estados de estrés y/o producir diversos efectos tóxicos. La capacidad de las plantas para acumular y tolerar los elementos tóxicos del medio, así como su movilidad, depende de las características físico-químicas del suelo, lo que afectará la disponibilidad del metal, su absorción y posterior translocación desde la raíz a los órganos aéreos,

Los efectos fitotóxicos que se observan luego de la exposición de algún elemento tóxico, incluyen inhibición del crecimiento, la clorosis, la falta de nutrientes y el estrés oxidativo (MÁRQUES-GARCÍA e CÓRDOVA, 2010; MORENO-JIMÉNEZ et al., 2008). Nuestros resultados, orientados a identificar vegetales acumuladoras de metales, han demostrado las potenciales características de las especies Atriplex atacamensis, Atriplex halimus, Lupinus microcarpus y Tessaria absinthioides, las cuales son componentes de las comunidades arbustivas desérticas del área pre-andina de la Región de Antofagasta, Chile (DÍAZ et al., 2011; TAPIA et al., 2016) y pueden extraer del suelo o acumular en sus raíces alguno de sus contaminantes, especialmente el As, metaloide de alta toxicidad y que se encuentra en altas concentraciones en esa región (QUEIROLO et al., 2000; DÍAZ et al., 2008).

En este contexto, el objetivo de esta revisión es informar acerca de la capacidad de acumulación y resistencia a ciertos elementos tóxicos del suelo para uso agrícola, de 4 especies vegetales que forman parte de la flora nativa de la Región de Antofagasta y de los efectos tóxicos del As, metaloide que se encuentra en altas concentraciones en L. microcarpus.

\section{Material y métodos}

\section{Área de Estudio}

Los lugares donde se recolectaron las muestras de suelos, se ubicaron en la zona central de Chile, Santiago, región Metropolitana (suelo de referencia) y áreas agrícolas de localidades pre-andinas cercanas a la ciudad de Calama, Región de Antofagasta, norte de Chile (suelo experimental) (Fig. 1). 


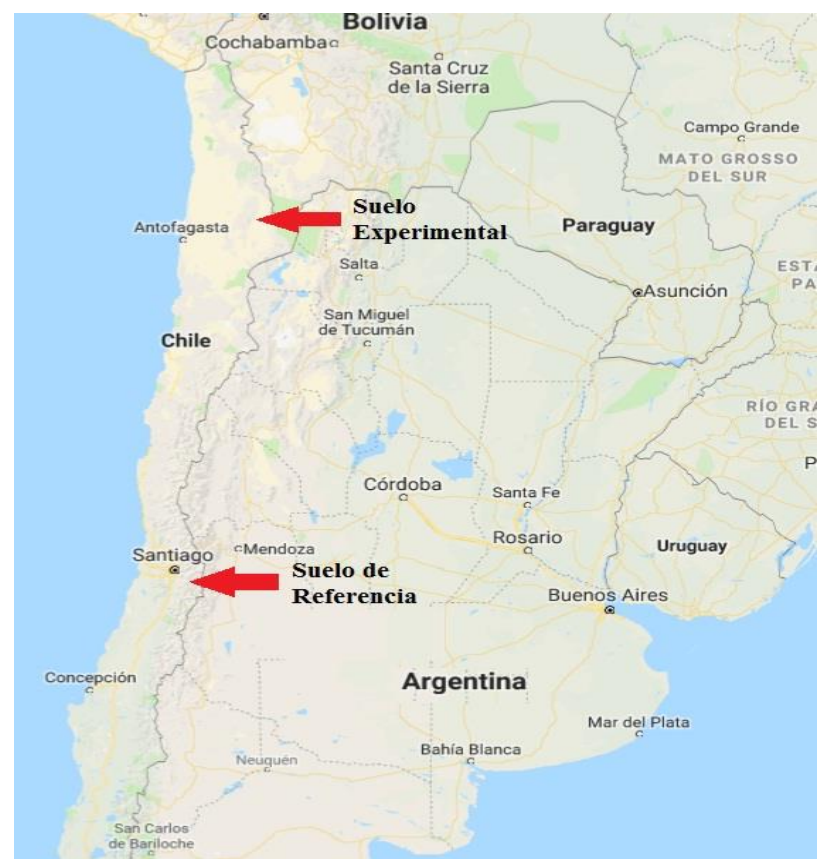

Figura 1. Área de estudio.

\section{Suelos y sus Propiedades Físico-químicas}

Muestras de suelo superficial $(0-15 \mathrm{~cm})$ fueron colectadas en áreas pre-andinas de zonas agrícolas cercanas a la ciudad de Calama y el río Loa, correspondiendo a los suelos experimentales del estudio. Producto de la escasez de lluvias y altas temperaturas durante el día, estos suelos son salinos y áridos (DÍAZ et al., 2016) y se caracterizan por presentar una escasa cubierta vegetal y una alta concentración de arsénico de origen natural (TAPIA et al., 2013). Las muestras de suelo de referencia fueron obtenidas en la zona central de Chile, suelos clasificados como arcilloso-limoso.

\section{Muestras Vegetales}

Vástagos de $A$. atacamensis y A. halimus fueron trasplantadas a maceteros de plásticos a los que se les agregó suelo procedente del norte de Chile (experimental) y de la zona central (de referencia). De cada planta se separaron los órganos aéreos (hojas y tallos) y las raíces (Tapia et al., 2013). Las especies seleccionadas en el estudio se muestran en la Figura 2.

Este procedimiento experimental se desarrolló durante 90 días y tuvo por objetivo analizar el grado de acumulación de elementos tóxicos del suelo, principalmente arsénico y el grado de resistencia de ambas especies vegetales a este elemento. Semillas geminadas de $L$. microcarpus fueron colocadas en maceteros de plástico conteniendo los suelos experimental y referencia (VÁSQUEZ et al., 2006; DÍAZ et al., 2016). Este procedimiento experimental se llevó a cabo por espacio de 180 días, en que mes a mes se controló el grado de desarrollo de las plantas y se observó la aparición de síntomas visuales de toxicidad (DÍAZ et al., 2016). Los ensayos tanto para A. atacamensis, A. halimus y L. microcarpus, se realizaron en el invernadero de la Universidad de Santiago de Chile (USACH), en condiciones ambientales controladas y protegidas de la lluvia. Las plantas se regaron 2 veces/semana con $200 \mathrm{~mL}$ de agua destilada por macetero. Durante los ensayos, la temperatura mínima promedio fue de $7.9 \pm 2.8^{\circ} \mathrm{C}$, 
mientras que la temperatura máxima alcanzó $23.4 \pm 4.6^{\circ} \mathrm{C}$. La humedad relativa fue de $60.3 \pm$ $26.5 \%$. Las especies Atriplex atacamensis, Tessaria absinthioides y Lupinus microcarpus fueron recogidas in situ en Lasana, Chiu Chiu y Ayquina, respectivamente, con el objetivo de determinar el grado de acumulación de arsénico en los distintos órganos, para lo cual se calculó el factor de bioconcentración (BCF) y el índice de transporte (TI) o factor de translocación de arsénico.

\section{Indicadores de estrés en especies del género Atriplex}

Indicadores de estrés en las especies del género Atriplex fueron determinados calculando el factor de bioconcentración, BCF (TU e MA, 2002), e índice de transporte, TI (RUIZ e ARMIENTA, 2012), midiendo la concentración de As por el método FI-HG-AAS (Flow Injection Hydride Generation_Atomic Absorption Spectrophotometry). La clorofila, los tioles totales y el malondialdehído (MDA), mediante espectrofotometría UV-visible, en cada órgano vegetal a comienzos del ensayo (día 0) y luego a los 30 y 90 días (MORENO-JIMÉNEZ et al., 2008; TAPIA et al., 2013).

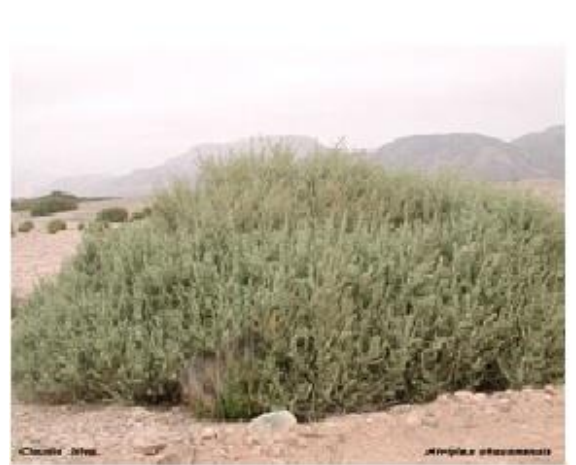

a)

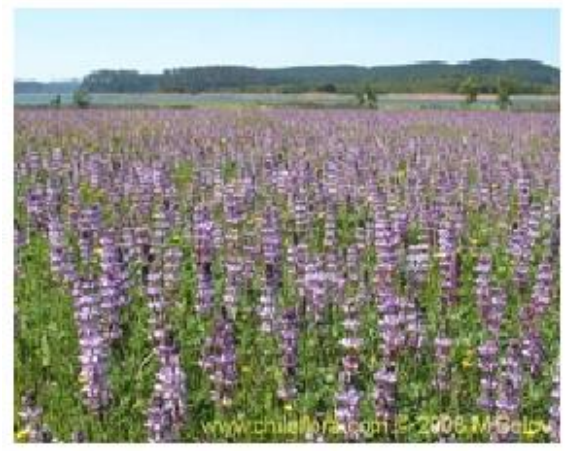

c)

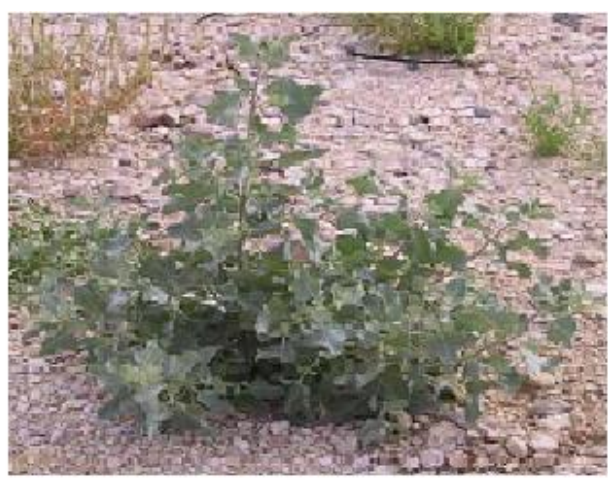

b)

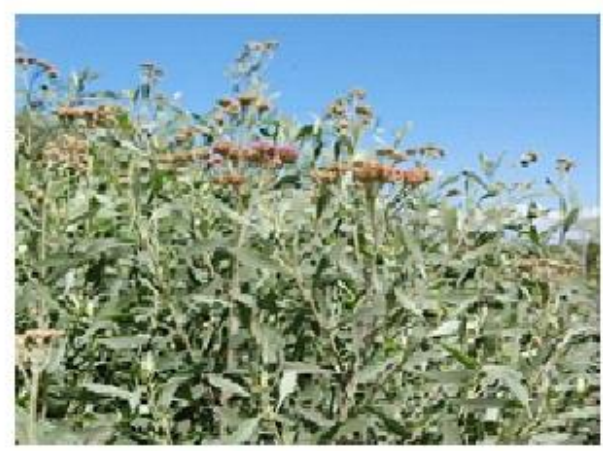

d)

Figura 2. Especies: a) Atriplex atacamensis, b) Atriplex halimus, c) Lupínus microcarpus y d) Tessaria absinthioides.

\section{Efectos tóxicos del arsénico en L. microcarpus}

Los síntomas visuales de toxicidad en L. microcarpus fueron observados y registrados cada mes, durante 6 , meses del comienzo de los experimentos, hojas y raíces fueron separadas 
y las partículas del suelo manualmente removidas para su análisis. (MUÑOZ et al., 2002; DÍAZ et al., 2011).

\section{Análisis estadístico de los datos}

El análisis estadístico de los datos se basó en un análisis de varianza (ANOVA) aplicado a los valores promedio de concentración de arsénico, clorofila, tioles totales, comprobando las diferencias estadísticamente significativas mediante el test de Duncan $(p \leq 0.05)$. Todos los análisis estadísticos se realizaron mediante software SPSS 13.0.

\section{Resultados y discusión}

\section{Características físico-químicas de los suelo}

Las características físico-químicas de los suelos experimental y de referencia, indicaron diferencias significativas de los contenidos de metales y metaloides, en la conductividad eléctrica y en la capacidad de intercambio catiónico (Tabla 1). Notable es la alta concentración de plomo en el suelo referencia. Además de la alta concentración de sodio presente en el suelo procedente del norte de Chile, lo cual junto con la alta conductividad eléctrica y bajo porcentaje en materia orgánica explican su marcada aridez; lo que junto a las altas concentraciones de arsénico y boro, elementos tóxicos, caracterizan a los suelos de la Región de Antofagasta.

Tabla 1. Propiedades físico-químicas del suelo experimental y suelo control (valores promedio \pm desviación estándar).

\begin{tabular}{lll}
\hline Característica & Suelo Experimental & Suelo Control \\
\hline $\mathrm{pH}^{\mathrm{a}}$ & $8.4 \pm 0.1$ & $7.8 \pm 0.1$ \\
$\mathrm{CE}^{\mathrm{a}}\left(\mathrm{dS} \mathrm{m}^{-1} 25^{\circ} \mathrm{C}\right)$ & $2.6 \pm 0.4$ & $1.7 \pm 0.2$ \\
$\mathrm{CIC}\left(\mathrm{cmol} \mathrm{kg}^{-1}\right)$ & $35 \pm 6.5$ & $19.0 \pm 4.5$ \\
Materia Orgánica (\%) & $0.81 \pm 0.1$ & $6.34 \pm 0.1$ \\
$\mathrm{~N}$ Kjeldahl (\%) & $0.21 \pm 0.01$ & $0.27 \pm 0.02$ \\
$\mathrm{As}\left(\mathrm{mg} \mathrm{kg}^{-1}\right)$ & $111 \pm 19$ & $12.7 \pm 1.1$ \\
$\mathrm{~B}\left(\mathrm{mg} \mathrm{kg}^{-1}\right)$ & $79.6 \pm 0.98$ & $1.13 \pm 0.06$ \\
$\mathrm{Cd}\left(\mathrm{mg} \mathrm{kg}^{-1}\right)$ & $<\mathrm{LD}$ & $<\mathrm{LD}$ \\
$\mathrm{Cu}\left(\mathrm{mg} \mathrm{kg}^{-1}\right)$ & $33.2 \pm 1.0$ & $431 \pm 21$ \\
$\mathrm{Na}\left(\mathrm{mg} \mathrm{kg}^{-1}\right)$ & $1263 \pm 72$ & $784 \pm 20$ \\
$\mathrm{P}$ total $(\%)$ & $0.06 \pm 0.01$ & $0.21 \pm 0.00$ \\
$\mathrm{~Pb}\left(\mathrm{mg} \mathrm{kg}^{-1}\right)$ & $<\mathrm{LD}$ & $302 \pm 109$ \\
$\mathrm{Zn} \mathrm{(mg} \mathrm{kg}$ & & $1700 \pm 52$ \\
Arcilla $(\%)$ & $43.2 \pm 4.1$ & 16 \\
Arena $(\%)$ & 13 & 50 \\
$\mathrm{Limo}(\%)$ & 64 & 34 \\
\hline
\end{tabular}

a $1: 5 \mathrm{w} / \mathrm{v}$ extracto en agua; LD: límite de detección; CE: conductividad eléctrica; CIC: capacidad de intercambio catiónico. 


\section{Grado de acumulación de arsénico en las especies $A$. atacamensis y A. halimus}

Los niveles de clorofila se mantuvieron en ambas especies cultivadas en el suelo experimental, que contiene altos niveles de arsénico y a una alta salinidad, respecto a las plantas que crecieron en el suelo de referencia (Fig. 3). Estudios previos indican que la concentración de clorofila en las hojas no es un buen indicador del estrés de una planta respecto a elementos del suelo, como el arsénico, posiblemente debido a su condición halofítica y al mecanismo de fotosíntesis C4 (AKHANI et al., 1997; MÁRQUEZ-GARCÍA e CÓRDOBA, 2010).

\section{Grado de resistencia}

Los niveles de tioles totales para cada especie de planta se mantuvieron constantes (Fig. 3), lo cual está de acuerdo con las mediciones en los órganos aéreos de plantas halofíticas, como es el caso del género Atriplex (LOLHANDE et al., 2011). Por otra parte, la concentración de MDA aumenta en $A$. halimus cultivada en suelo recogido en el norte de Chile debido a su alta concentración de As y probablemente de boro en este suelo, elementos de conocidos efectos fitotóxicos (TAPIA et al., 2013; DÍAZ et al., 2016). El estrés oxidativo, se ve reflejado por el aumento en la producción de malondialdehído (MDA), mientras que el grupo -SH (tiol), puede anular el estrés oxidativo mediante la formación de complejos (MORENO-JIMÉNEZ et al., 2008; TAPIA et al., 2013).
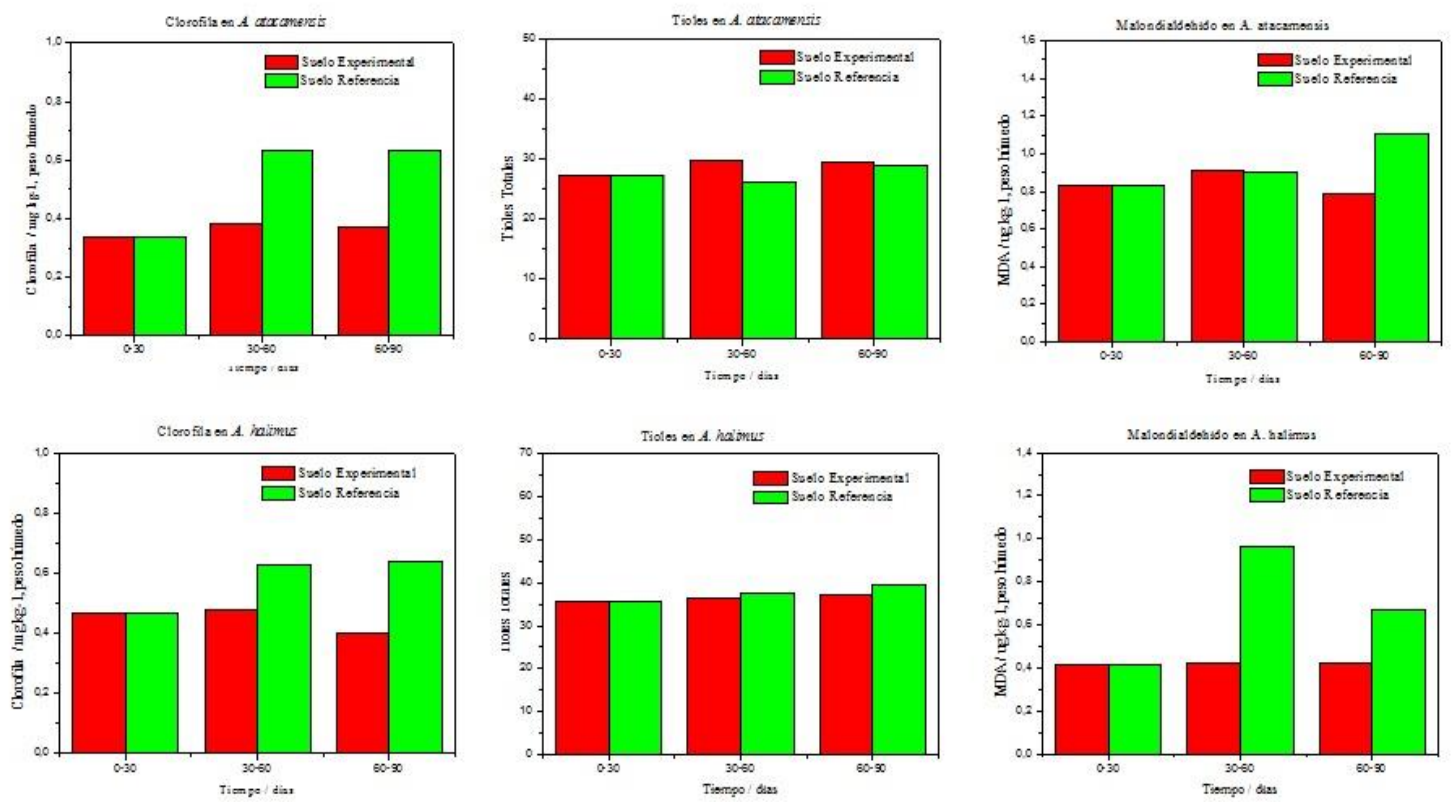

Figura 3. Grado de acumulación de As y resistencia al As de la especies Atriplex atacamensis y Atriplex halimus.

\section{Síntomas de toxicidad en la especie Lupinus microcarpus}

La Figura 4 muestra los resultados de los síntomas visuales de toxicidad en la especie $L$. microcarpus. Las plantas que crecieron en los suelos del norte de Chile, mostraron todos los síntomas visuales de toxicidad: clorosis foliar, necrosis del ápice y bordes de las hojas, además 
de hojas arrugadas y un marcado decrecimiento al final del ensayo. Estos síntomas significaron la muerte de todas las plantas al final del estudio. Por otra parte, todas las plantas que crecieron en suelo referencia, no experimentaron ninguno de los síntomas de toxicidad indicados.

Estos efectos son atribuibles a una restricción de los movimientos del agua en la planta, generando estrés hídrico, el cual finalmente significa la muerte del vegetal (MARÍN et al., 1992; CARBONELL-BARRACHINA et al., 1997). Esta situación no se observa en especies de plantas resistentes al arsénico, como se indicó anteriormente en el caso de las especies $A$. atacamensis y $A$. halimus, las cuales crecieron normalmente y no evidenciaron síntomas visuales de toxicidad. Se debe asumir que las plantas resistentes al arsénico compartimentalizan y/o transforman el arsénico en otras especies arsenicales menos fitotóxicas, en orden a resistir la alta carga del metaloide en la célula (MEHARG e HARTLEY-WHITAKER, 2002). La Tabla 2 muestra las concentraciones de arsénico en las muestras de suelo, hojas y raíces de la especie L. microcarpus en desarrollo y los valores de BCF y TI.
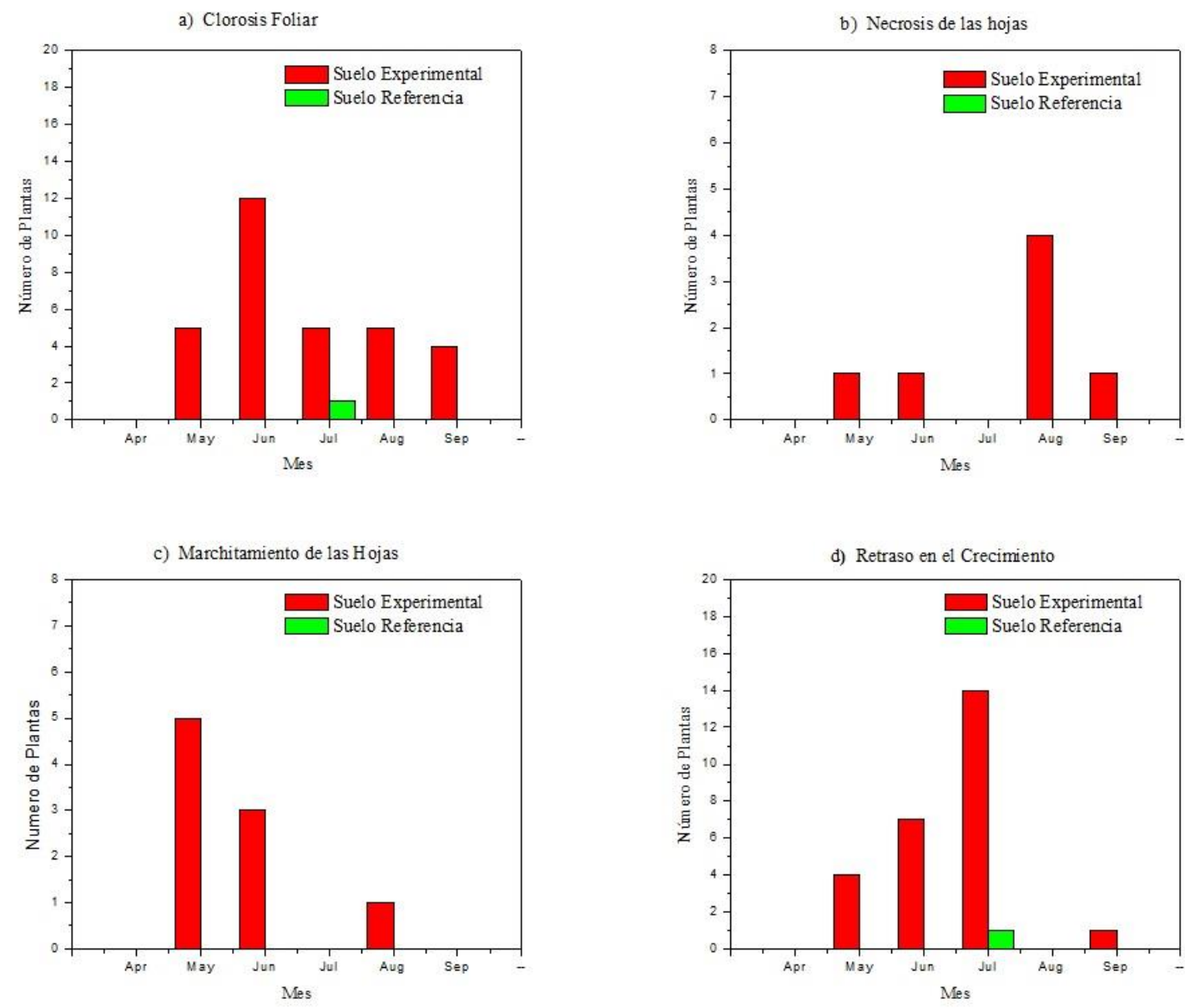

Figura 4. Efectos tóxicos del As en la especie Lupinus microcarpus. 
Grado de acumulación de arsénico en las especies $A$. atacamensis, A. halimus, L. microcarpus y T. absinthioides

La mayor concentración de arsénico en las hojas, se encontró en la especie $L$. microcarpus, resultando significativa la diferencia respecto a los niveles medidos en el tallo y raíz. El suelo en el cual se colectó la planta mostró la concentración más baja del elemento (5.6 $\mathrm{mg} \mathrm{kg}^{-1}$ ), respecto a la concentración de arsénico medida en las muestras de suelo donde crecieron las otras especies.

El índice de transporte (IT) es una importante forma de medir la capacidad de acumular metal de un vegetal y su posterior aplicación en técnicas de fitorremediación (BAKER e WHITING, 2002; SUN et al., 2008). Los valores sobre 1.0 obtenidas por las especies $L$. microcarpus y $T$. absinthioides indican que éstas muestran una gran capacidad de bioacumulación y translocación del metaloide, en especial la especie L. microcarpus, respecto a los obtenidos en las otras especies vegetales analizadas. Como los altos valores de BCF indican que la fitoextracción de cualquier elemento del suelo es factible (Mc GRATH e ZAO, 2003), los resultados de la concentración de As en hojas y raíz en L. microcarpus cultivado experimentalmente en suelo con bajos niveles del elemento ratifican a esta especie como una buena acumuladora de arsénico (BCF $=1.80)$. En ese caso, se obtuvo valores inferiores de As en hojas y raíz, porque los individuos no completaron su ciclo de vida, debido posiblemente a una acción sinérgica entre la acción fitotóxica del As y la alta salinidad del suelo, como lo determinó Carbonell-Barrachina et al. (1997).

Tabla 2. Concentración de As en hojas, tallos y raíces de A. atacamensis, A. halimus, L. microcarpus y $T$. absinthioides adultos. Valores de BCF e TI.

\begin{tabular}{l|l|l|l|l|l|l}
\hline \multirow{2}{*}{ Especie } & \multicolumn{4}{l}{ Concentración de As $\left(\mathrm{mg} \mathrm{kg}^{-1}\right.$, peso seco $)$} & \multirow{2}{*}{ BCF } & \multirow{2}{*}{$\mathrm{TI}$} \\
\cline { 2 - 7 } & Suelo & Hojas & Tallo & Raíz & & \\
\hline A. atacamensis & $111 \pm 19$ & $2.6 \pm 0.7$ & $3.2 \pm 0.9$ & $6.9 \pm 1.2$ & 0.02 & 0.4 \\
A. halimus & $111 \pm 19$ & $5.6 \pm 1.9$ & $4.7 \pm 0.5$ & $16.3 \pm 3.8$ & 0.05 & 0.5 \\
L. microcarpus & $5.6 \pm 1.0$ & $9.7 \pm 1.6$ & $2.5 \pm 0.9$ & $1.6 \pm 0.4$ & 1.80 & 6.1 \\
T. absinthioides & $53 \pm 9.9$ & $5.9 \pm 1.0$ & $5.1 \pm 0.4$ & $3.4 \pm 0.2$ & 0.10 & 1.7 \\
\hline
\end{tabular}

\section{Conclusiones}

Los suelos agrícolas del desierto árido de Chile en la Región de Antofagasta, muestran una alta salinidad, bajo contenido de materia orgánica y alta concentración de metales y metaloides, algunos de ellos en concentraciones que los hacen altamente tóxicos.

Las especies $A$. atacamensis y $A$. halimus crecieron sin síntomas visuales de toxicidad y sin crecimiento reducido en suelos áridos y con alta concentración de arsénico. La clorofila y los tioles no fueron afectados negativamente y solamente $A$. halimus mostró un aumento en los niveles de MDA. 
Los valores de $\mathrm{Tl}$ indican que las especies A. halimus y L. microcarpus acumularon altos niveles de arsénico en la parte área, lo cual debiera ser considerado en el caso de que estas plantas sean usadas en alimentación animal. A su vez, la especie L. microcarpus tiende a concentrar el arsénico en la raíz, en la primeras etapas de desarrollo, y en la medida que crece el metaloide es translocado a las hojas.

A. atacamensis y $A$. halimus resisten la alta salinidad del suelo y los altos niveles de arsénico y probablemente de otros elementos, pudiéndose recomendar en programas de cubrimiento vegetal de suelos áridos en la zona pre-andina de Antofagasta. A su vez, A. halimus y L. microcarpus pueden ser recomendados para aplicar programas de fitorremediación de suelos agrícolas con altos niveles de arsénico y niveles naturales y de baja salinidad. En estas condiciones, estas especies se comportan como fitoestabilizadoras y fitoextractoras de As en suelos agrícolas, respectivamente.

\section{Agradecimientos}

La información presentada ha sido financiada por el Programa Iberoamericano de Ciencia y Tecnología para el Desarrollo (CYTED) de España, el Programa Agrícola de la Corporación del Cobre (CODELCO), División Chuquicamata, el Instituto de Agroquímica y Tecnología de Alimentos (IATA-CSIC) de Valencia, España, el Proyecto FONDECYT 3110001 y el Proyecto 4091466 de la Dirección de Gestión Tecnológica (DGT), perteneciente a la Universidad de Santiago de Chile (USACH).

\section{Referencias}

1. AKHANI, H.; TRIMBORN, P.; ZIEGLER, H. Phytosynthetic path ways in Chenopodiaceae from Africa, Asia, and Europe with their ecological, phytogeographical and taxonomical importance. Plant Systematic and Evolution, v. 206, p. 187-221, 1997.

2. BAKER, A.J.M.; WHITING, S.N. In search of the holy grail - a further step in understanding metal hyper accumulation? New Phytologist, v. 155, p. 1-7, 2002.

3. CARBONELL-BARRACHINA, A.A.; BURLÓ-CARBONELL, F.; MATAIX-BENEYTO, J. Arsenic uptake, distribution and accumulation in bean plants: Effect of arsenite and salinity on plant growth and yield. Journal of Plant Nutrition, v. 20, p. 1419-1430, 1997.

4. DÍAZ, O.; PASTENE, R.; RECABARREN, E.; NÚÑEZ, N.; VÉLEZ, D.; MONTORO, R. Arsenic contamination from geological sources in environmental compartments in pre-Andean area of northern Chile. In: BUNSCHUH, J.; ARMIENTA, M.A.; BHATTACHARYA, P.; MATSCHULLAT, J.; MUKKERJEE, B. (Eds.) Natural Arsenic in Groundwater of Latin America-Occurrence, Health Impact and Remediation. Taylor and Francis Group, p. 335344, 2008.

5. DÍAZ, O.; TAPIA, Y.; PASTENE, R.; MONTES, S.; NÚÑEZ, N.; VÉLEZ, D.; MONTORO, R. Total and bioavailable arsenic concentration in arid soils and its uptake by native plants from the pre-Andean zones in Chile. Bulletin of Environmental Contamination and Toxicology, v. 86, p. 666-669, 2011. 
6. DÍAZ, O.; TAPIA, Y.; PASTENE, R.; CAZANGA, M.; SEGURA, R.; PEREDO, S. (2016). Lupinus microcarpus growing in arsenic - agricultural soils from Chile: Toxic effects and it potential use as phytorremediator plant. Journal of Environmental Protection, v. 7, n. 1, p. 116-128, 2016.

7. LASAT, M.M. The use of plants for the removal of toxic metals from contaminated soil. American Association for the Advancement of Science. Environmental Science and Engineering Fellow, 33 p. 2000.

8. LOLHANDE, V.H.; SRIVASTAVA, S.; PATADE, V.Y.; DWIVEDI, S.; TRIPATHI, R.D.; NIKAM, T.D. Investigation of arsenic accumulation and tolerance potential of Sesuvium portulacastrum (L). Chemosphere, v. 82, p. 529-534, 2011.

9. MARÍN, A.R.; PEZESHKI, S.R.; MASSCHELEYN, P.H.; CHOI, H.S. The influence of chemical form and concentration of arsenic in rice growth and tissue arsenic concentration. Plant and Soil, v. 139, p. 175-183, 1992.

10. MÁRQUEZ-GARCÍA, B., CÓRDOVA, F. Antioxidative system in wild populations of Erica andevalensis. Environmental and Experimental Botany, v. 68, p. 58-65, 2010.

11. Mc GRATH, S.P.; ZHAO, F.J. Phytoextraction of metals and metalloids from contaminated soils. Current Opinion in Biotechnology, v. 14, p. 277-282, 2003.

12. MEHARG, A.A.; HARTLEY-WHITAKER, J. Arsenic uptake and metabolism in arsenic resistant and nonresistant plant species. New Phytologist, v. 54, n. 1, p. 29-43, 2002.

13. MORENO-JIMÉNEZ, E.; PEÑALOSA, J.; CARPENA, R.; ESTEBAN, E. Comparison of arsenic resistance in Mediterranean woody shrubs used in restoration activities. Chemosphere, v. 71, p. $466-473,2008$

14. MUÑOZ, O.; DÍAZ, O.; LEYTON, I.; NÚÑEZ, N.; DEVESA, V.; SUÑER, M.; VÉLEZ, D.; MONTORO, R. Vegetables collected in the cultivated Andean área of Northern Chile: Total and inorganic arsenic contents in raw vegetables. Journal of Agricultural and Food Chemistry, v. 50, p. 642-647, 2002.

15. QUEIROLO, F.; STEGEN, S.; MONDACA, J.; CORTÉS, R.; ROJAS, R.; CONTRERAS, C.; MUNOZ, L.; SCHWUGER, M.J.; OSTAPCZUK, P. Total arsenic, lead, cadmium, copper, and zinc in some salt rivers in the northern Andes of Antofagasta, Chile. Science of the Total Environment, v. 255, n. 1-3, p. 85-95, 2000.

16. RUIZ, E.A.; ARMIENTA M.A. Acumulación de arsénico y metales pesados en maízn en suelos cercanos a jales o residuos mineros. Revista Internacional de Contaminación Ambiental, v. 28, n. 2, p. 103-117, 2012. 
17. SUN, Y.; ZHOU, Q.; DIAO, C.H. Effects of cadmium and arsenic on growth and metal accumulation of Cd-hyperaccumulator Solanum nigrum L. Bioresource Technology, v. 99, p. 1103-1110, 2008.

18. TAPIA, Y.; DÍAZ, O.; PIZARRO, C.; SEGURA, R.; VINES, H.; ZÚÑIGA, G.; MORENO-JIMÉNEZ, E. Atriplex atacamensis and Atriplex halimus resist arsenic contamination in pre- Andean soils (northern Chile). Science of the Total Environment, v. 450-451, p. 188-196, 2013.

19. TAPIA, Y.; DÍAZ, O.; ACUÑA, E.; CASANOVA, M.; SALAZAR, O.; MASAGUES, A. Phytostabilization of arsenic in soils with plants of the genus Atriplex established in situ in the Atacama Desert. Environmental Monitoring and Assessment, v. 188, n. 4, p. 1-11, 2016.

20. TU, C.; MA, L.Q. Effects of Arsenic Concentrations and Forms on Arsenic Uptake by the Hyperaccumulator Ladder Brake. Journal of Environmental Quality Abstract - Plant and Environment Interactions, v. 31, n. 2, p. 641-647, 2002.

21. VÁSQUEZ, S.; AGHA, R.; GRANADO, A.; SARRO, M.J.; ESTEBAN, E.; PEÑALOSA, J.M.; CARPENA, R.O. Use of white lupin plant for phytostabilization of $\mathrm{Cd}$ and As polluted acid soil. Water, Air and Soil Pollution, v. 177, p. 349-365, 2006. 